\title{
The impact of COVID-19 on human reproduction and directions for fertility treatment during the pandemic
}

\author{
Dayong Lee \\ Department of Obstetrics and Gynecology, Kyungpook National University School of Medicine, Daegu, Korea
}

Since December 2019, severe acute respiratory syndrome coronavirus 2 (SARS-CoV-2) has spread rapidly, resulting in a pandemic. The virus enters host cells through angiotensin-converting enzyme 2 (ACE2) and transmembrane protease serine subtype 2 (TMPRSS2). These enzymes are widely expressed in reproductive organs; hence, coronavirus disease 2019 (COVID-19) could also impact human reproduction. Current evidence suggests that sperm cells may provide an inadequate environment for the virus to penetrate and spread. Oocytes within antral follicles are surrounded by cumulus cells, which rarely express ACE2 and TMPRSS2. Thus, the possibility of transmission of the virus through sexual intercourse and assisted reproductive techniques seems unlikely. Early human embryos express coronavirus entry receptors and proteases, implying that human embryos are potentially vulnerable to SARS-CoV-2 in the early stages of development. Data on the expression of ACE2 and TMPRSS2 in the human endometrium are sparse. Moreover, it remains unclear whether SARS-CoV-2 directly affects the embryo and its implantation. A study of the effect of SARS-CoV-2 on pregnancy showed an increase in preterm delivery. Thus, vertical transmission of the virus from mother to fetus in the third trimester is possible, and further data on human reproduction are required to establish this possibility. Based on analyses of existing data, major organizations in this field have published guidelines on the treatment of infertility. Regarding these guidelines, despite the COVID-19 pandemic, reproductive treatment is crucial for the well-being of society and must be continued under suitable regulations and good standard laboratory practice protocols.

Keywords: COVID-19; Pregnancy; Reproduction; SARS-CoV-2

\section{Introduction}

In December 2019, a novel infectious disease was reported in China, and the World Health Organization defined this viral illness as coronavirus disease 2019 (COVID-19). This disease is mainly a respiratory infection with symptoms including fever, cough, and shortness of breath. Severe acute respiratory syndrome coronavirus 2 (SARSCoV-2) is the viral pathogen that causes COVID-19. Coronaviruses are

Received: March 16, 2021 · Revised: May 13, 2021 · Accepted: June 10, 2021 Corresponding author: Dayong Lee

Department of Obstetrics and Gynecology, Kyungpook National University School of Medicine, 130 Dongdeok-ro, Jung-gu, Daegu 41944, Korea

Tel: +82-53-200-5724 Fax: +82-53-423-7905 E-mail: wabhappy@knu.ac.kr

This is an Open Access article distributed under the terms of the Creative Commons Attribution Non-Commercial License (http://creativecommons.org/licenses/by-nc/4.0/) which permits unrestricted non-commercial use, distribution, and reproduction in any medium, provided the original work is properly cited. enveloped, single-stranded ribonucleic acid (RNA) viruses that express a characteristic corona-like morphology under an electron microscope. In the last 2 decades, humanity has suffered three major coronavirus outbreaks - severe acute respiratory syndrome coronavirus (SARS-CoV), Middle East respiratory syndrome coronavirus (MERS-CoV), and SARS-CoV-2 - that have evoked considerable worldwide health consternation [1-3]. The differences between these three coronaviruses and the patterns of their outbreaks are summarized in Table 1.

SARS-CoV-2 is mainly transmitted via respiratory droplets, although viral RNA has been detected in various samples, such as nasal secretions, sputum, feces, and rarely in the serum and urine [4]. SARS-CoV-2 has a very effective binding affinity to angiotensin-converting enzyme 2 (ACE2) in human cells. The coronavirus invades the cell by attaching the viral spike (S) glycoprotein to ACE2 and employing the cellular serine protease (transmembrane protease serine 
Table 1. Biological and clinical characteristics of SARS, MERS, and COVID-19

\begin{tabular}{llll}
\hline & & & MERS (2012) \\
\hline Pathogen & SARS-CoV & MERS-CoV & COVID-19 \\
Reservoir & Bat-musk cat & Bat-camel & Bat-pangolin \\
Target enzyme & ACE2 & DPP4 & ACE2 \\
Case & $8,096[1]$ & $2,519[1]$ & $>161,000,000^{\mathrm{a})}$ \\
Death & $774(9.6 \%)[1]$ & $866(34.3 \%)[1]$ & $>3,343,000(2.1 \%)^{\mathrm{a})}$ \\
Mode of transmission & Droplets & Droplets & Droplets, limited evidence of other routes \\
Incubation period (day) & $4-7$ & $2-14$ & $1-14$ \\
Key symptom & Cough, fever, diarrhea & Fever, cough, shortness of breath & Fever, cough, shortness of breath \\
At-risk group & People with underlying medical conditions & Men $>60 \mathrm{yr}$ & Adults $>60$ yr with underlying medical \\
& & & conditions \\
Treatment & No specific & No specific & No specific \\
Vaccine & No & No & Yes \\
\hline
\end{tabular}

SARS, severe acute respiratory syndrome; MERS, Middle East respiratory syndrome; COVID-19, coronavirus disease 2019; CoV, coronavirus; ACE2, angiotensinconverting enzyme 2; DPP4, dipeptidyl peptidase-4.

${ }^{\text {a) }}$ Figures as of May 2021.

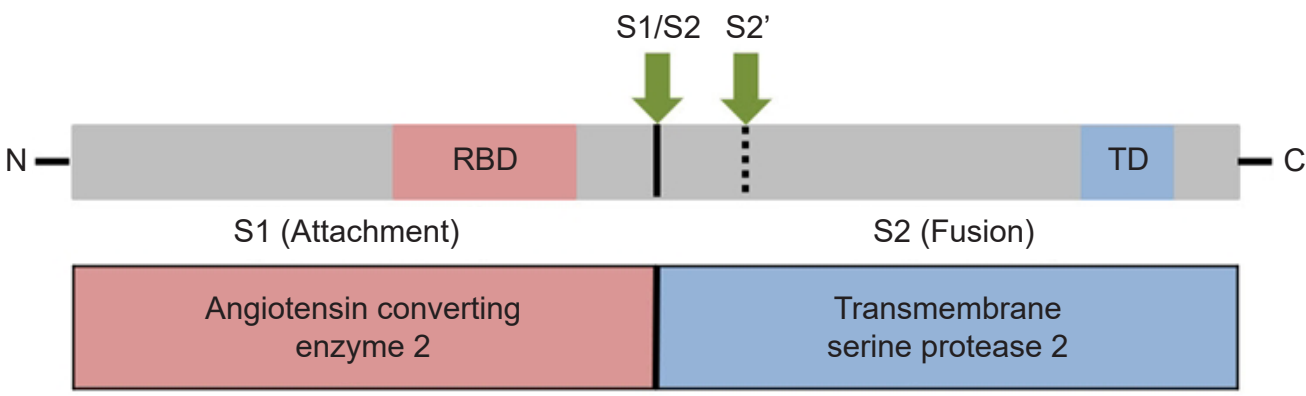

Figure 1. Overview of the spike glycoprotein of severe acute respiratory syndrome coronavirus 2 (SARS-CoV-2) and target proteins. Functional domains: receptor binding domain (RBD), transmembrane domain (TD). Proteolytic cleavage sites: S1/S2, S2'.

subtype 2 [TMPRSS2]) to cleave the viral S protein to provoke fusion of the viral and host cell membranes. These enzymes are expressed in a wide range of human organs, including reproductive organs and respiratory tissues [5].

Cardiac, nasal, ocular, and neurological symptoms of COVID-19 have been reported. However, the impact of COVID-19 on the human reproductive system remains debatable and warrants investigation. It is important to establish whether natural and assisted reproduction can be safely accomplished in the era of the COVID-19 pandemic. Thus, the purpose of this review was to investigate how SARSCoV- 2 affects the various stages of human reproduction through available research on the subject.

\section{Sex differences in the transmission of SARS-CoV-2}

The $S$ glycoprotein of the coronavirus is the key protein present on the outer envelope of the virion and plays a principal role in viral entrance by recognizing host cell receptors and modulating fusion of the viral and cellular membranes [6]. The $S$ protein is synthesized and then cleaves to the $\mathrm{S} 1$ subunit of the $\mathrm{N}$ terminal and the $\mathrm{S} 2$ subunit of the $\mathrm{C}$ terminal (Figure 1).

Viral entry into the host requires the SARS-CoV2 S protein to bind to the host ACE2 receptor. A host protease, TMPRSS2, acts to cleave the viral $S$ protein at the host cell membrane. Through this process, priming cleavage of the S protein between $\mathrm{S} 1$ and $\mathrm{S} 2$ takes place, followed by activating cleavage at the $\mathrm{S} 2$ site. Coexpression of ACE2 and TMPRSS2, which is crucial for SARS-CoV-2 infection, has been observed in various tissue types, including the lung, nose, heart, coIon, and kidney, where COVID-19 related symptoms have been reported [7].

It is plausible that SARS-CoV2 infection might have a detrimental effect on human reproductive function if cells of the male or female reproductive organs also express these genes. Limited data suggest that the receptor basigin/CD147 (BSG) may induce viral entry, similarly to ACE2 [8], and the cysteine protease cathepsin L (CTSL) can potentially cleave to the viral S protein, similarly to TMPRSS2 [9]. Recently, Shilts et al. [10] reported that recombinant SARS-CoV-2 S proteins do not interact with BSG expressed on the membrane of human cells; therefore, the role of these alternate entry receptors is not definitive. 
Studies have suggested that females infected with COVID-19 have lower morbidity, mortality, and severe case rates than males, as well as better outcomes. Ding et al. [11] reported that non-menopausal female patients had milder symptoms and better prognoses than age-matched male patients. Among female patients, menopausal women had a longer hospitalization period than non-menopausal women. In that study, both anti-Müllerian hormone (AMH) and estradiol (E2) levels showed significant negative correlations with disease severity. Specifically, E2 levels were negatively correlated with inflammatory markers including interleukin (IL)-2R, IL-6, IL-8, and tumor necrosis factor-alpha in the luteal phase and C3 in the follicular phase. The authors speculated that female hormones in premenopausal women may act as protective factors against COVID-19. Sex differences in the disease severity and prognosis were previously attributed to sex-dependent production of steroid hormones, different copy numbers of immune response $X$-linked genes, and the presence of disease susceptibility genes, such as TLR7, IFNB1, and IL6 [12]. These results suggest that female hormones in premenopausal women may provide a protective effect against COVID-19. Khan [13] speculated that $17 \beta-E 2$ would decrease SARS-CoV-2 infection by regulating the renin-angiotensin-aldosterone system, suppressing the inflammatory storm, inducing antiviral immune responses, and promoting the degradation of the virus in endolysosomes. One research group reported that premenopausal women taking combined oral contraceptives had a significantly lower predicted COVID-19 positivity with a reduction in the duration of hospital stay [14]. In another recent study, Ding et al. [15] reported a significant decrease in AMH levels after SARS-CoV-2 infection, suggesting that ovarian injury may occur through COVID-19. Therefore, the causal relationship between the severity of the disease and levels of $\mathrm{AMH}$ and estrogen should be carefully considered, and more clinical studies investigating this issue should be conducted.

Cell type-specific expression of genes that produce viral host entry proteins and identification of potential loci of viral infection within the reproductive system are essential to predict whether SARS-CoV-2 has the potential to affect natural and assisted human reproduction. Through studies conducted on gametes, embryos, endometrium, and placenta, we investigated the possible effects of SARS-CoV-2 on human reproduction.

\section{Effects of SARS-CoV-2 on testes and male gametes}

The presence of ACE2 and TMPRSS2 in reproductive organs is considered a precondition for susceptibility to SARS-CoV-2 infection. Stanley et al. [16] analyzed publicly available single-cell RNA sequencing (scRNA-seq) datasets, their transcriptomic data, and publicly available bulk RNA and proteomics data on human and nonhuman primate reproductive organ tissues. They analyzed the cell-type-specific expression pattern of ACE2 and TMPRSS2. The expression of alternative receptor BSG and protease CTSL were also evaluated. The 11 cell types of the human testes, including somatic niche cells (Leydig cells, endothelial cells, myoid cells, and macrophages) and germ cells (differentiating spermatogonia, early primary spermatocytes, late primary spermatocytes, round spermatids, elongated spermatids, and sperm) were analyzed. A small proportion of spermatogonial stem cells expressed ACE2 and TMPRSS2, although cells coexpressing both genes were extremely rare ( $0.05 \%$ of cells). This low rate of coexpression was similar in other testicular cell types, although the researchers were not able to explore Sertoli cells. These results suggest that sperm cells may not be at increased risk of viral invasion and spread through ACE2 and TMPRSS2. The alternative receptor BSG and protease CTSL were generally expressed in testicular cell types. However, it has not yet been proven whether CTSL can effectively replace TMPRSS2 in viral S protein priming.

Testicular pain and discomfort have been observed in some male patients infected with COVID-19 [17]. Compensated hypogonadism with no statistically significant difference in serum testosterone and follicle-stimulating hormone levels, but significantly higher serum luteinizing hormone levels, has also been reported [18]. These symptoms and signs suggest that SARS-CoV-2 infection may affect the testes. Although SARS-CoV-2 was detected in the testis by reverse transcription-polymerase chain reaction in one case [19], it is still unclear whether SARS-CoV-2 can directly penetrate the testis because there is no additional direct evidence of this possibility. However, in that study, a postmortem examination of the testes of 12 patients revealed significant seminiferous tubular injury, reduced Leydig cells, and mild lymphocytic inflammation.

SARS-CoV-2 may disrupt spermatogenesis through multiple pernicious mechanisms that include febrile conditions and oxidative stress. Febrile conditions can decrease the concentration of sperm and increase the proportion of abnormal sperm [20]. SARS-CoV-2 infection activates oxidant-sensitive pathways via inflammatory responses, and reactive oxygen species are produced during this process [21]. This stress stimulates the release of cytokines, causing an exaggeration of the inflammatory responses. Holtmann et al. [22] reported that men who recovered from moderate COVID-19 infection showed a statistically significant impairment in semen parameters compared with men who recovered from mild symptoms and the control group. The detection of SARS-CoV-2 RNA in semen was reported by Li et al. [23], though the methodology of sample collection appears flawed and there was a possibility of contamination. In other studies, no virus was detected in semen samples [22,24,25].

These results suggest that long-term effects of SARS-CoV-2 on male reproductive function are unlikely, and the possibility of transmission of the virus from men through sexual intercourse and assist- 
ed reproductive techniques (ART) is low, although further research is required on this subject.

\section{Effects of SARS-CoV-2 on ovaries and female gametes}

To date, there are insufficient data on scRNA in human ovaries, specifically the outer ovarian cortex. Published scRNA-seq datasets on nonhuman primate ovarian tissue including oocytes and six somatic cell types (stromal cells, granulosa cells, smooth muscle cells, natural killer cells, macrophages, and endothelial cells) were analyzed [16]. According to these data, a subpopulation of oocytes in nonhuman primate ovarian tissue coexpressed ACE2 and TMPRSS2, while coexpression was not observed in ovarian somatic cells. The coexpression of ACE2 and TMPRSS2 in oocytes increased according to follicular maturation: primordial follicles expressed minimal coexpression, while $62 \%$ of antral follicles had detectable coexpression. Considering that antral follicles either undergo ovulation or regression within several days of the follicular phase in each menstrual cycle, a sustained impact of the virus on oocytes seems unlikely. A transcriptomic and proteomic analysis by Virant-Klun and Strle [26] also revealed that human oocytes expressed both genes and proteins for ACE2 and BSG. Essahib et al. [27] visualized the viral entry protein by immunohistochemistry using a donated human oocyte. In their study, BSG was mainly present on the oolemma, while ACE2 was not expressed in primary oocytes.

The transcriptome analysis of human cumulus cells using novel RNA-seq datasets revealed that ACE2 is widely expressed in these cell types. By contrast, the expression of TMPRSS2 was reported to be absent or extremely low in human cumulus cells, which is concordant with the primate scRNA-seq results. Therefore, cumulus cells may serve as a physical barrier, and oocytes enclosed with cumulus cells are unlikely to be susceptible to SARS-CoV-2 infection. However, the virus may affect oocytes in other ways, such as through oxidative stress, inflammatory cytokines, distorted metabolism, and changes within the ovarian follicle or ovarian cortical tissues.

\section{Effects of SARS-CoV-2 on embryos}

Available research results on human gametes indicate that SARSCoV-2 infection may not have a long-term effect on male and female reproductive function. Although this statement cannot be interpreted as a definitive conclusion, it suggests that there is a low risk of viral transmission from gametes to embryos during ART, including oocyte retrieval and in vitro fertilization. Although there is currently no evidence that SARS-CoV-2 is transmitted through coitus, ART minimizes the risk of exposing reproductive cells to viral infection compared to natural conception.

Some viruses can infect developing embryos. For example, the Zika virus can infect and propagate into the trophectoderm cells of a preimplantation human embryo, causing apoptosis of neural progenitor cells, microcephaly, and miscarriage [28]. Because SARSCoV-2 is highly contagious, and a significant number of infected individuals are asymptomatic, certain women are likely to become pregnant during the course of subclinical infection. This acts as a risk not only for women who are pregnant naturally, but also for women who are pregnant through ART.

Analysis of publicly available scRNA-seq datasets of zygotes, fourcell and eight-cell embryos, morulae, inner cell masses, epiblasts, primitive endoderms, and trophectoderms for the coronavirus receptors (ACE2, BSG), and the $S$ protein cleavage enzymes (TMPRSS2, CTSL) showed that ACE2 and BSG are expressed in the cells from the zygote to blastocyst stages, including the trophectodermal lineage [29]. ACE2, TMPRSS2, BSG, and CTSL are coexpressed in some proportion of epiblast cells and most trophectoderm cells. Moreover, the embryonic and trophectodermal cells also express genes for viral replication, interaction with SARS-CoV-2, and endosomal sorting complexes required for transport. Similarly, Weatherbee et al. [30] showed the expression and coexpression of ACE and TMPRSS2 genes in the trophoblast of the blastocyst and syncytiotrophoblast, as well as in the hypoblast, which develop into placental tissues that interact with the maternal blood supply for oxygen and nutrient exchange. Visualizing viral receptors through immunohistochemistry revealed that in preimplantation and peri-implantation blastocysts, ACE2 and BSG were present on the membrane of trophectoderm and hypoblast cells, which will both develop into the embryonic part of the placenta (chorion). Both receptors were also present on the membrane of epiblast cells, which will differentiate into the embryo [27].

These results imply that SARS-CoV-2 is theoretically able to bind and invade human pre- and peri-implantation embryos. Both embryonic and extra-embryonic cell lineages display these receptors. This indicates that the virus could invade the embryo at distinct time points before, during, and after implantation through vertical transmission. Further research is needed to determine whether viral infection occurs in vivo or whether it causes failure of implantation, miscarriage, pregnancy complications, fetal compromise, and/or congenital malformations.

\section{Effects of SARS-CoV-2 on the endometrium and implantation}

The effects of SARS-CoV-2 on endometrial tissue and implantation remain inconclusive. In this situation, to determine the potential susceptibility of the endometrium to infection by SARS-CoV- 2 and possible damage thereafter, Henarejos-Castillo et al. [31] assessed SARSCoV-2 infection-related gene expression of ACE2, TMPRSS2, TMPRSS4, BSG, cathepsin B (CTSB), CTSL, FURIN, and MX1 from endometrial transcriptomic data sets. The results revealed that TMPRSS4 increased viral susceptibility on its own, and this was observed in the 
epithelial cells of gut tissue [32].

Transcriptomic data sets available across the phases of an endometrial cycle were analyzed to evaluate the molecular risk of SARSCoV-2 infection. Gene expression data on normal endometrium from five studies were assembled throughout the menstrual cycle and included samples from the proliferative phase, early secretory phase, mid-secretory phase, and late secretory phase. ACE2, the principal receptor for SARS-CoV-2 cell entry, showed low expression in the endometrium. The expression of ACE2 increased (fold change, 2.47) from the early secretory to mid-secretory phases, implying an increase of ACE2 expression during the window of implantation and a higher risk of viral infectivity at this stage. TMPRSS2, the major protease involved in SARS-CoV-2 cleavage together with ACE2, had medium endometrial expression throughout the menstrual cycle. There was no correlation between TMPRSS2 and ACE2. These results imply that the endometrium is safe against SARS-CoV-2 infection. Other minor proteases such as TMPRSS4, CTSL, CTSB, and furin showed high expression during certain periods of the menstrual cycle, although none of the highly expressed proteases in this study were reported to initiate SARS-CoV-2 infection. The positive correlation between age and ACE2 from the proliferative to mid-secretory phases, especially in the early secretory phase, suggests that the endometrium in older women could be more susceptible to SARS-CoV-2 infection. However, no prospective studies have assessed the real-world clinical implications of this possibility, and verification is needed.

Overall, the endometrium appears to have low susceptibility to SARS-CoV-2 infection based on low ACE2 and TMPRSS2 expression. Further research on endometrial tissue of women of reproductive age infected with SARS-CoV-2 will help determine whether the causal implications deduced from molecular characterization of the endometrium translates to actual clinical manifestations.

\section{Effects of SARS-CoV-2 on the placenta and pregnancy}

SARS-CoV-2 has the potential to affect the embryo. During the early developmental stage, the cells of the embryo are enclosed by a layer of trophectodermal cells that differentiate to form the placenta. ACE2 and TMPRSS2 are highly expressed in the developing trophectoderm and human placental cells. This suggests that the early stage of a placenta may be permissive to viral infection through vertical transmission of SARS-CoV-2 during early pregnancy via maternal blood. Indeed, virions were detected in the trophoblast cells of the placenta in pregnant women infected with COVID-19 [33-35]. Furthermore, placental damage was observed in pregnancies complicated by SARS-CoV-2 infection [36,37]. However, there are little experimental and clinical data concerning the actual risks of vertical transmission at this earlier stage of pregnancy. Likewise, there is a dearth of information on maternal and neonatal outcomes after
SARS-CoV-2 infection in the first to second trimester of pregnancy.

An existing systematic review and meta-analysis on pregnancy and neonatal outcomes reported an increased risk of preterm birth, preeclampsia, cesarean section, and perinatal mortality if COVID-19 infection occurs in the early third trimester [38,39]. Consistently, a Centers for Disease Control and Prevention report on the U.S. population showed that among 3,912 infants born to mothers with SARSCoV-2 infection, $12.9 \%$ were preterm ( $<37$ weeks), which is higher than the US national estimate of $10.2 \%$ [40]. Among neonates with test results, $2.6 \%$ tested positive for SARS-CoV-2, and they were born primarily to mothers who were infected at delivery. The occurrence of preterm birth did not differ by the status of maternal symptoms. With regard to pregnancy outcomes, $99.3 \%$ had a live birth, $0.3 \%$ miscarried before 20 weeks, and $0.4 \%$ miscarried after 20 weeks. These study results are compared with those of previous coronavirus infections (SARS-CoV and MERS-CoV) in Table 2 [3,38-41].

Conflicting clinical reports have been published regarding SARSCoV-2 infection in neonates born to mothers infected with SARSCoV-2 before childbirth. Some studies reported no evidence of vertical transmission in this situation, while others found that some neonates were born with the SARS-CoV-2 infection [42,43]. However, the methods of sample detection or processing in many studies were flawed. Some studies failed to detect SARS-CoV-2 in neonates or only reported the presence of specific antibodies associated with the viral infection. Others detected the virus, but the route of transmission was not clear, as maternal or newborn blood, amniotic fluid, and placenta were not thoroughly investigated in order to ascertain the maternal and neonatal relationship. Besides, in many of these cases, the possibility that secondary infection occurred immediately after birth among the caregivers cannot be excluded.

A case report describing transplacental transmission of SARSCoV-2 in a neonate born to a mother infected at the end of the third trimester ( $35+5$ weeks of gestational age) suggests that vertical transmission may be possible at this stage [44]. In this case, (1) maternal viremia; (2) placental infection demonstrated by immunohistochemistry and a high viral load, and placental inflammation confirmed by histological examination and immunohistochemistry; and (3) neonatal viremia following placental infection were confirmed. These comprehensive virological and pathological investigations strongly suggest vertical infection. The neonate presented with neurological manifestations indicated by white matter injury, gradually recovered, and was subsequently discharged from the hospital. Based on this case, transplacental transmission may be possible in the last weeks of pregnancy. Nonetheless, possible transmission and fetal consequences in the first or second trimester are not definitive, and further studies focusing on long-term outcomes in these cases are required. 
Table 2. Pregnancy outcomes of SARS, MERS, and COVID-19

\begin{tabular}{|c|c|c|c|}
\hline & SARS (2002) & MERS (2012) & COVID-19 \\
\hline Case fatality rate & $15 \%[41]$ & $23 \%[41]$ & $1 \%[39]$ \\
\hline \multicolumn{4}{|c|}{ Pregnancy outcome (reported cases per pregnancy) } \\
\hline Miscarriage & $67 \%(8 / 12)[3]$ & NA & $<1 \%[40]$ \\
\hline \multirow[t]{2}{*}{ Preterm delivery at $<37$ weeks } & $33 \%(5 / 15)[3]$ & $27 \%(3 / 11)[3]$ & $26 \%(98 / 386)[39]$ \\
\hline & & & $11 \%(506 / 4,442)[40]$ \\
\hline Preterm delivery at $<34$ weeks & $27 \%(4 / 15)[3]$ & $27 \%(3 / 11)[3]$ & $3 \%(149 / 4,442)[40]$ \\
\hline \multirow[t]{2}{*}{ Cesarean delivery } & $78 \%(7 / 9)[3]$ & $63 \%(5 / 8)[3]$ & $93 \%(38 / 41)[3]$ \\
\hline & & & $34 \%(1,331 / 2,589)[40]$ \\
\hline \multicolumn{4}{|c|}{ Neonatal outcome (reported cases per neonate) } \\
\hline Apgar score $<7$ & $0 \%(0 / 4)[3]$ & $0 \%(0 / 3)[3]$ & $2 \%(1 / 41)[3]$ \\
\hline Neonatal death & $0 \%(0 / 9)[3]$ & $30 \%(3 / 10)[3]$ & $1 \%(4 / 369)[39]$ \\
\hline Vertical transmission & No case & No case & Possible \\
\hline
\end{tabular}

SARS, severe acute respiratory syndrome; MERS, Middle East respiratory syndrome; COVID-19, coronavirus disease 2019; NA, not applicable.

Table 3. Effect of COVID-19 on each component of human reproduction

\begin{tabular}{|c|c|}
\hline Component & Major effect \\
\hline \multirow[t]{2}{*}{ Male gamete } & Rare co-expression of ACE2 and TMPRSS2 \\
\hline & Sperm cells may not be at an increased risk of viral invasion and spread. \\
\hline \multirow[t]{3}{*}{ Female gamete } & Increased co-expression of ACE2 and TMPRSS2 according to follicular maturation \\
\hline & Very low or no expression of TMPRSS2 on human cumulus cells \\
\hline & Oocytes enclosed with cumulus cells are unlikely to be susceptible to infection. \\
\hline \multirow[t]{2}{*}{ Embryo } & Co-expression of ACE and TMPRSS2 in the developing embryo \\
\hline & Possible viral infection; however, further evidence is required. \\
\hline \multirow[t]{2}{*}{ Endometrium } & Low ACE2 and TMPRSS2 expression \\
\hline & Assumed low susceptibility to SARS-CoV-2 infection; however, further research is needed. \\
\hline \multirow[t]{2}{*}{ Placenta } & High expression of ACE2 and TMPRSS2 in developing placenta \\
\hline & Vertical transmission through placenta in third trimester is possible; however, this has not been confirmed in the first or second trimester. \\
\hline
\end{tabular}

COVID-19, coronavirus disease 2019; ACE2, angiotensin-converting enzyme 2; TMPRSS2, transmembrane protease serine subtype 2; SARS-CoV-2, severe acute respiratory syndrome coronavirus 2 .

\section{Conclusions}

Through a review of the existing published studies, the possible effects of COVID-19 on human reproduction are summarized in Table 3. With regard to human gametes, sperm cells may not be at an increased risk of viral entry and spread. Oocytes within antral follicles are surrounded by cumulus cells, which rarely express ACE2 and TMPRSS2. Thus, a sustained impact of the SARS-CoV-2 on oocytes also seems unlikely. These results suggest that infertility treatment can be performed safely if human gametes are obtained and manipulated in line with the established principles for preventing contamination in ART. Additional studies and data are needed to determine the possibility of SARS-CoV-2 affecting the endometrium during the implantation period or the embryo in the early stages of development, and thereby interfering with implantation or increasing the rate of miscarriage. Since the probability of preterm delivery or pregnancy-re- lated complications increases when a pregnant woman is infected with SARS-CoV-2, more emphasis should be placed on preventing infection in these women. However, additional studies on how asymptomatic or self-limiting infection during pregnancy may affect long-term pregnancy outcomes or fetal and neonatal health should be conducted. Nevertheless, it is necessary to be cautious when considering the causal relationships in existing research findings, and the results of molecular biological and clinical studies that are actively being conducted in this area should be regularly reviewed.

Over the last year, accumulated data on molecular virology, immunobiology, and clinical manifestations of SARS-CoV-2 infection have improved the understanding of the effect of this novel disease on human reproduction. As knowledge of the infection increased, the guidelines of major academic societies on the implementation of ART also changed. The guidelines published by major organizations on human reproduction are listed in chronological order and sum- 
marized in Table 4 [45-51]. In the early stages of the COVID-19 pandemic, the effects of SARS-CoV-2 on ART or pregnancy outcomes were unclear, and these concerns suggested that ART should be postponed except for emergent cases. However, one of the most important factors in determining fertility is the woman's age, and the more deferred the infertility treatment, the worse the outcomes of ART. Moreover, the detrimental effects of SARS-CoV-2 infection increase in severity with age, and this fact implies that older women of reproductive age undergoing ART could be at higher risk of severe symptoms and worsened ART and pregnancy outcomes. Accordingly, the time variable is crucial in specific subgroups of infertile couples, particularly women with a diminished ovarian reserve who tend to lose their fertility potential rapidly with an expected poor prognosis for ART.

In light of the rapidly accumulated knowledge and consensus that infertility treatment should not remain suspended for a long time for the well-being of infertile couples and the health of society, ART should be maintained despite the pandemic. Regardless of the clinical indications, ART should resume under established regulations for local circumstances and characteristics. Given the fact that SARSCoV-2 has high surface stability on various materials, there are many potential routes by which cross-contamination of reproductive tissue within the laboratory can take place. Patients should be screened before any procedures, and active COVID-19 infections and suspected

Table 4. Representative international guidelines for infertility treatment during the COVID-19 pandemic

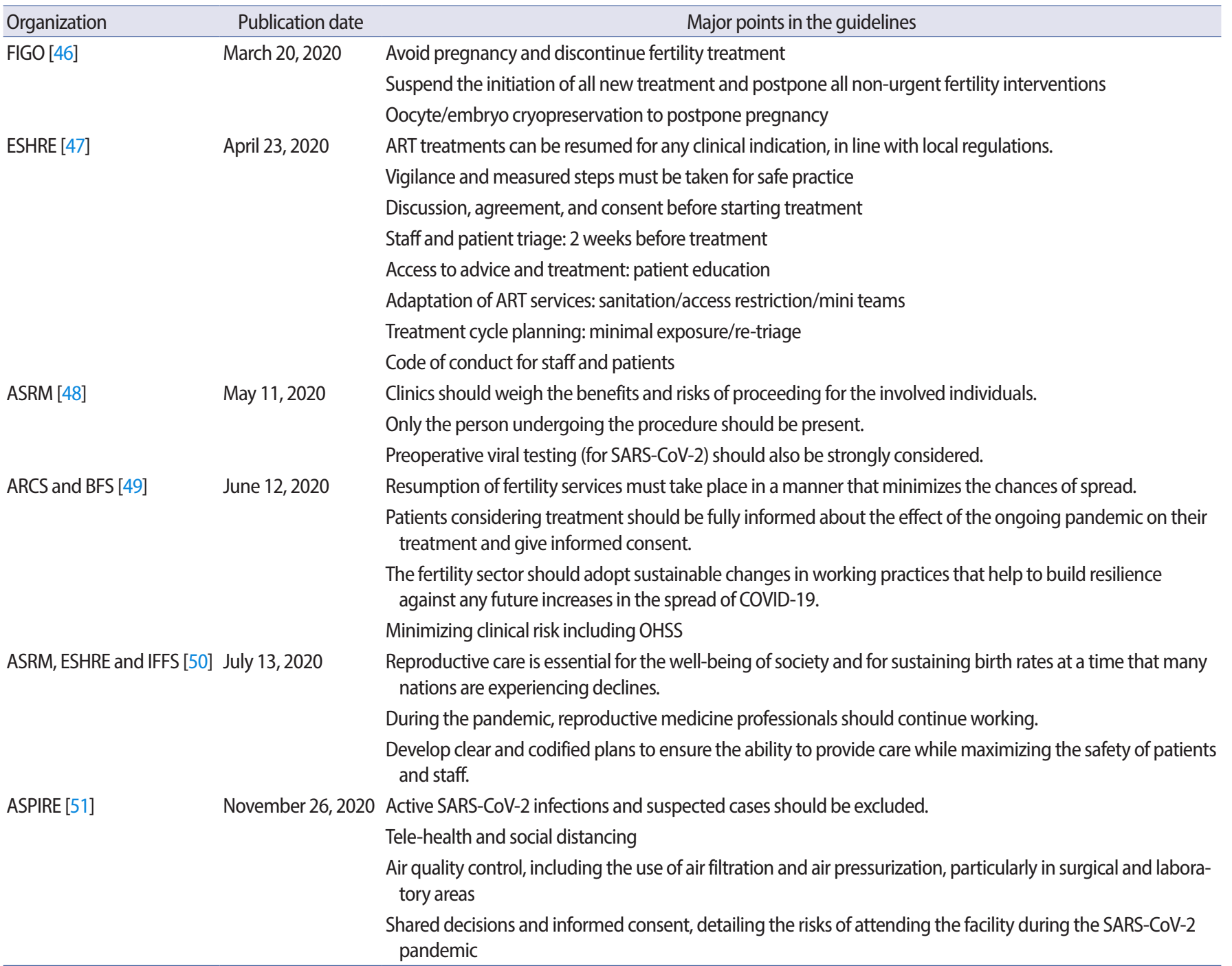

COVID-19, coronavirus disease 2019; FIGO, International Federation of Gynecology and Obstetrics; ESHRE, European Society of Human Reproduction and Embryology; ART, assisted reproductive techniques; ASRM, American Society for Reproductive Medicine; SARS-CoV-2, severe acute respiratory syndrome coronavirus 2; ARCS, Association of Reproductive and Clinical Scientists; BFS, British Fertility Society; OHSS, ovarian hyperstimulation syndrome; IFFS, International Federation of Fertility Societies; ASPIRE, Asia Pacific Initiative on Reproduction. 
patients should be excluded. Both patients and healthcare providers should adhere to the use of appropriate personal protective equipment designated for each phase of the ART procedure. Infertility treatment laboratories manipulating gametes and embryos should establish and strictly follow good standard laboratory practices.

\section{Conflict of interest}

No potential conflict of interest relevant to this article was reported.

\section{ORCID}

\section{Dayong Lee}

https://orcid.org/0000-0003-4340-8180

\section{References}

1. da Costa VG, Moreli ML, Saivish MV. The emergence of SARS, MERS and novel SARS-2 coronaviruses in the 21 st century. Arch Virol 2020;165:1517-26.

2. Peeri NC, Shrestha N, Rahman MS, Zaki R, Tan Z, Bibi S, et al. The SARS, MERS and novel coronavirus (COVID-19) epidemics, the newest and biggest global health threats: what lessons have we learned? Int J Epidemiol 2020;49:717-26.

3. Di Mascio D, Khalil A, Saccone G, Rizzo G, Buca D, Liberati M, et al. Outcome of coronavirus spectrum infections (SARS, MERS, COVID-19) during pregnancy: a systematic review and meta-analysis. Am J Obstet Gynecol MFM 2020;2:100107.

4. Holshue ML, DeBolt C, Lindquist S, Lofy KH, Wiesman J, Bruce H, et al. First case of 2019 novel coronavirus in the United States. N Engl J Med 2020;382:929-36.

5. Hoffmann M, Kleine-Weber H, Schroeder S, Kruger N, Herrler T, Erichsen S, et al. SARS-CoV-2 cell entry depends on ACE2 and TMPRSS2 and is blocked by a clinically proven protease inhibitor. Cell 2020;181:271-80.

6. Shang J, Ye G, Shi K, Wan Y, Luo C, Aihara H, et al. Structural basis of receptor recognition by SARS-CoV-2. Nature 2020;581:221-4.

7. Goyal P, Choi JJ, Pinheiro LC, Schenck EJ, Chen R, Jabri A, et al. Clinical characteristics of COVID-19 in New York city. N Engl J Med 2020;382:2372-4.

8. Wang K, Chen W, Zhang Z, Deng Y, Lian JQ, Du P, et al. CD147spike protein is a novel route for SARS-CoV-2 infection to host cells. Signal Transduct Target Ther 2020;5:283.

9. Yan R, Zhang Y, Li Y, Xia L, Guo Y, Zhou Q. Structural basis for the recognition of SARS-CoV-2 by full-length human ACE2. Science 2020;367:1444-8.

10. Shilts J, Crozier TW, Greenwood EJ, Lehner PJ, Wright GJ. No evi- dence for basigin/CD147 as a direct SARS-CoV-2 spike binding receptor. Sci Rep 2021;11:413.

11. Ding T, Zhang J, Wang T, Cui P, Chen Z, Jiang J, et al. Potential influence of menstrual status and sex hormones on female severe acute respiratory syndrome coronavirus 2 infection: a cross-sectional multicenter study in Wuhan, China. Clin Infect Dis 2021;72: e240-8.

12. Channappanavar R, Fett C, Mack M, Ten Eyck PP, Meyerholz DK, Perlman S. Sex-based differences in susceptibility to severe acute respiratory syndrome coronavirus infection. J Immunol 2017;198: 4046-53.

13. Khan N. Possible protective role of $17 \beta$-estradiol against COVID-19. J Allergy Infect Dis 2020;1:38-48.

14. Costeira R, Lee KA, Murray B, Christiansen C, Castillo-Fernandez J, Ni Lochlainn M, et al. Estrogen and COVID-19 symptoms: associations in women from the COVID Symptom Study. PLoS One 2021; $16: \mathrm{e} 0257051$

15. Ding T, Wang T, Zhang J, Cui P, Chen Z, Zhou S, et al. Analysis of ovarian injury associated with covid-19 disease in reproductive-aged women in Wuhan, China: an observational study. Front Med (Lausanne) 2021;8:635255.

16. Stanley KE, Thomas E, Leaver M, Wells D. Coronavirus disease-19 and fertility: viral host entry protein expression in male and female reproductive tissues. Fertil Steril 2020;114:33-43.

17. Kim J, Thomsen T, Sell N, Goldsmith AJ. Abdominal and testicular pain: an atypical presentation of COVID-19. Am J Emerg Med 2020;38:1542.

18. Ma L, Xie W, Li D, Shi L, Mao Y, Xiong Y, et al. Effect of SARS-CoV-2 infection upon male gonadal function: a single center-based study. MedRxiv 20037267 [Preprint]. 2020 [cited 2021 Nov 1]. Available from: https://doi.org/10.1101/2020.03.21.20037267.

19. Yang M, Chen S, Huang B, Zhong JM, Su H, Chen YJ, et al. Pathological findings in the testes of COVID-19 patients: clinical implications. Eur Urol Focus 2020;6:1124-9.

20. Abdelhamid MH, Walschaerts M, Ahmad G, Mieusset R, Bujan L, Hamdi S. Mild experimental increase in testis and epididymis temperature in men: effects on sperm morphology according to spermatogenesis stages. Transl Androl Urol 2019;8:651-65.

21. Delgado-Roche L, Mesta F. Oxidative stress as key player in severe acute respiratory syndrome coronavirus (SARS-CoV) infection. Arch Med Res 2020;51:384-7.

22. Holtmann N, Edimiris P, Andree M, Doehmen C, Baston-Buest D, Adams O, et al. Assessment of SARS-CoV-2 in human semen-a cohort study. Fertil Steril 2020;114:233-8.

23. Li D, Jin M, Bao P, Zhao W, Zhang S. Clinical characteristics and results of semen tests among men with coronavirus disease 2019. JAMA Netw Open 2020;3:e208292. 
24. Song C, Wang Y, Li W, Hu B, Chen G, Xia P, et al. Detection of 2019 novel coronavirus in semen and testicular biopsy specimen of COVID-19 patients. MedRxiv 20042333 [Preprint]. 2020 [cited 2021 Nov 1]. Available from: https://doi.org/10.1101/2020.03.31. 20042333

25. Fan C, Li K, Ding Y, Lu WL, Wang J. ACE2 expression in kidney and testis may cause kidney and testis damage after 2019-nCoV infection. MedRxiv 20022418 [Preprint]. 2020 [cited 2021 Nov 1]. Available from: https://doi.org/10.1101/2020.02.12.20022418.

26. Virant-Klun I, Strle F. Human oocytes express both ACE2 and BSG genes and corresponding proteins: is SARS-CoV-2 infection possible? Stem Cell Rev Rep 2021;17:278-84.

27. Essahib W, Verheyen G, Tournaye H, Van de Velde H. SARS-CoV-2 host receptors ACE2 and CD147 (BSG) are present on human oocytes and blastocysts. J Assist Reprod Genet 2020;37:2657-60.

28. Tan L, Lacko LA, Zhou T, Tomoiaga D, Hurtado R, Zhang T, et al. Preand peri-implantation Zika virus infection impairs fetal development by targeting trophectoderm cells. Nat Commun 2019;10: 4155.

29. Colaco S, Singh K, Zhou N, Bhide A, Singh D, Singh A, et al. Expression of SARS-CoV-2 receptor ACE2 and the spike protein processing enzymes in developing human embryos. arXiv 2020;2004. 04935.

30. Weatherbee BA, Glover DM, Zernicka-Goetz M. Expression of SARS-CoV-2 receptor ACE2 and the protease TMPRSS2 suggests susceptibility of the human embryo in the first trimester. Open Biol 2020;10:200162.

31. Henarejos-Castillo I, Sebastian-Leon P, Devesa-Peiro A, Pellicer A, Diaz-Gimeno P. SARS-CoV-2 infection risk assessment in the endometrium: viral infection-related gene expression across the menstrual cycle. Fertil Steril 2020;114:223-32.

32. Zang R, Gomez Castro MF, McCune BT, Zeng Q, Rothlauf PW, Sonnek NM, et al. TMPRSS2 and TMPRSS4 promote SARS-CoV-2 infection of human small intestinal enterocytes. Sci Immunol 2020; 5:eabc3582.

33. Singh M, Bansal V, Feschotte C. A single-cell RNA expression map of human coronavirus entry factors. Cell Rep 2020;32:108175.

34. Li M, Chen L, Zhang J, Xiong C, Li X. The SARS-CoV-2 receptor ACE2 expression of maternal-fetal interface and fetal organs by single-cell transcriptome study. PLoS One 2020;15:e0230295.

35. Ashary N, Bhide A, Chakraborty P, Colaco S, Mishra A, Chhabria K, et al. Single-cell RNA-seq Identifies cell subsets in human placenta that highly expresses factors driving pathogenesis of SARSCoV-2. Front Cell Dev Biol 2020;8:783.

36. Bahadur G, Homburg R, Yoong W, Singh C, Bhat M, Kotabagi P, et al. Adverse outcomes in SAR-CoV-2 (COVID-19) and SARS virus related pregnancies with probable vertical transmission. JBRA As- sist Reprod 2020;24:351-7.

37. Verma S, Carter EB, Mysorekar IU. SARS-CoV2 and pregnancy: an invisible enemy? Am J Reprod Immunol 2020;84:e13308.

38. Knight M, Bunch K, Vousden N, Morris E, Simpson N, Gale C, et al. Characteristics and outcomes of pregnant women admitted to hospital with confirmed SARS-CoV-2 infection in UK: national population based cohort study. BMJ 2020;369:m2107.

39. Gajbhiye RK, Modi DN, Mahale SD. Pregnancy outcomes, newborn complications and maternal-fetal transmission of SARSCoV-2 in women with COVID-19: a systematic review of 441 cases. MedRxiv 20062356 [Preprint]. 2020 [cited 2021 Nov 1]. Available from: https://doi.org/10.1101/2020.02.12.20062356.

40. Woodworth KR, Olsen EO, Neelam V, Lewis EL, Galang RR, Oduyebo T, et al. Birth and infant outcomes following laboratory-confirmed SARS-CoV-2 infection in pregnancy: SET-NET, 16 Jurisdictions, March 29-October 14, 2020. MMWR Morb Mortal Wkly Rep 2020;69:1635-40.

41. Segars J, Katler Q, McQueen DB, Kotlyar A, Glenn T, Knight Z, et al. Prior and novel coronaviruses, coronavirus disease 2019 (COVID-19), and human reproduction: what is known? Fertil Steril 2020;113: 1140-9.

42. Kotlyar AM, Grechukhina O, Chen A, Popkhadze S, Grimshaw A, Tal O, et al. Vertical transmission of coronavirus disease 2019: a systematic review and meta-analysis. Am J Obstet Gynecol 2021;22 4:35-53.

43. Yang Z, Liu Y. Vertical transmission of severe acute respiratory syndrome coronavirus 2: a systematic review. Am J Perinatol 2020; 37:1055-60.

44. Vivanti AJ, Vauloup-Fellous C, Prevot S, Zupan V, Suffee C, Do Cao J, et al. Transplacental transmission of SARS-CoV-2 infection. Nat Commun 2020;11:3572.

45. Tolu LB, Feyissa GT, Jeldu WG. Guidelines and best practice recommendations on reproductive health services provision amid COVID-19 pandemic: scoping review. BMC Public Health 2021;21: 276.

46. FIGO Committee Reproductive Medicine, Endocrinology and Infertility. Fertility treatment and COVID-19 - April 2020 guidance [Internet]. London: International Federation of Gynecology and Obstetrics; 2020 [cited 2021 Mar 16]. Available from: https://www. figo.org/fertility-treatment-and-covid-19-april-2020-guidance.

47. ESHRE COVID-19 Working Group. ESHRE guidance on recommencing ART treatments [Internet]. Grimbergen: European Society of Human Reproduction and Embryology; 2020 [cited 2021 Mar 16]. Available from: https://www.eshre.eu/Home/COVID19WG.

48. American Society for Reproductive Medicine. Patient management and clinical recommendations during the coronavirus 
(COVID-19) pandemic [Internet]. Birmingham: American Society for Reproductive Medicine; 2020 [cited 2021 Mar 16]. Available from: https://www.asrm.org/news-and-publications/covid-19/ statements/patient-management-and-clinical-recommendations-during-the-coronavirus-covid-19-pandemic.

49. ARCS/BFS COVID Working Group. The Association of Reproductive and Clinical Scientists (ARCS) and British Fertility Society (BFS) U.K. best practice guidelines for reintroduction of routine fertility treatments during the COVID-19 pandemic [Internet]. Brentford: British Fertility Society; 2020 [cited 2021 Mar 16]. Available from: https://www.britishfertilitysociety.org.uk/2020/06/12/arcs-andbfs-u-k-best-practice-guidelines-for-reintroduction-of-routinefertility-treatments-during-the-covid-19-pandemic-2.

50. Ory S, Veiga A, Horton M, Gianaroli L. Joint IFFS/ESHRE statement on COVID-19 vaccination for pregnant women and those considering pregnancy. Hum Reprod Open 2021;2021:hoab016.

51. Wiweko B, Ho TM, Li R, Li TC, Tzeng CR, Kovacs G, et al. SARS-CoV-2 and assisted reproductive technology practice: an Asia Pacific Initiative on Reproduction (ASPIRE) position paper. Fertil Reprod 2020;2:115-43. 